Voix et Images

voixetimages

\title{
La poésie comme une passion tranquille
}

\section{Jacques Paquin}

Volume 23, numéro 2 (68), hiver 1998

La censure 1920-1960

URI : https://id.erudit.org/iderudit/201377ar

DOI : https://doi.org/10.7202/201377ar

Aller au sommaire du numéro

\section{Éditeur(s)}

Université du Québec à Montréal

\section{ISSN}

0318-9201 (imprimé)

1705-933X (numérique)

Découvrir la revue

\section{Citer cet article}

Paquin, J. (1998). La poésie comme une passion tranquille. Voix et Images, 23(2), 409-413. https://doi.org/10.7202/201377ar d'utilisation que vous pouvez consulter en ligne.

https://apropos.erudit.org/fr/usagers/politique-dutilisation/ 


\title{
La poésie comme une passion tranquille
}

\author{
Jacques Paquin, Université du Québec à Trois-Rivières
}

Le plus récent recueil de Nicole Brossard, Vertige de l'avant-scène ${ }^{1}$, s'inscrit dans la continuité des recueils précédents comme Langues obscures $^{2}$ et Installations $^{3}$. Le propos n'est pas nouveau, sans pour autant être répétitif, c'est d'ailleurs le propre de la grande poésie. Le plaisir de l'écriture que s'attache à transmettre Nicole Brossard depuis ses tout débuts se conjugue à un souci de lisibilité, et la pensée, fruit du travail du poème, y gagne d'autant en clarté. "Je m'intéresse à la connaissance" (p. 11), écrivait-elle dans Langues obscures. Penser, c'est une manière de bouger, et préférablement avec lenteur, avec souplesse, avec flexibilité, l'un des mots clés qu'on retrouve avec insistance dans Vertige de l'avant-scène. La main se place, se déplace, celle de l'écriture comme celle du désir, tout aussi interchangeables. La pensée passe par la fiction, mais il est difficile de ne pas sentir la présence diffuse d'un sujet empirique, jusque dans les moments où la poète, écrivant "je suis ", nous rappelle l'illusion du sujet en poésie. Toute pensée porte donc en elle sa fiction et toute fiction porte en elle sa pensée - "dans la fiction explorer la vérité" (p. 43).

Malgré sa mise en évidence par l'intitulé, le vertige n'apparaît qu'une seule fois au sein du recueil, il est une sensation éprouvée au quotidien et qui fait déboucher sur l'éternité. Est-ce la peur du vide qui fait que nous soyons avides d'images? Nous sommes avides de sensations, nous dit Brossard, colletés dans l'étroitesse et le fugace, en regard de l'immensité, de l'éternel et de la permanence, qui forment une configuration propre à aggraver le sentiment de la précarité. Ce vertige, s'il était énoncé avec pathos, ou s'il mimait les dérives du pulsionnel, placerait le sujet trop en avant-scène. Dans ce contexte, le sujet n'est pas exclu, mais demeure à ras de texte, bien qu'il puisse devenir à son tour objet de langue et, en particulier, d'une langue qu'il faut encore traduire:

la littérature est façon d'être une manière de traduire je suis toucher là où d'autres existent (p. 29) 
L'attraction du vide se manifeste, sur le plan de l'économie du langage, par une prosodie qui emprunte à la forme de la spirale, figure qui constitue une marque reconnaissable de l'écriture brossardienne. À deux reprises, l'immensité ouvre et boucle un texte de chacune des sections du recueil. Et à chaque fois, lorsqu'elle est près de l'éprouver, est-ce à cause des rumeurs, toujours désespérantes, à l'origine de "la manie de mal lire" (p. 7), le sujet est forcé de revenir sur ses pas, de trouver un autre chemin, de préférence le plus long. L'utopie paraît à portée de la main, on rêve de pouvoir raconter son enfance au futur, le mythe de l'avantdire est toujours très fort, même dans une écriture aussi consciente d'ellemême.

Le recueil est composé de deux parties, l'une en vers, l'autre en prose, donnant lieu à une partition (au double sens du terme) créatrice de corrélations favorisées par l'usage de deux registres. La première partie, dont l'intitulé coiffe le recueil, est la plus importante. Elle semble travaillée par le désir de la conversation qui est la forme la plus spontanée et la plus souple de la parole. L'écriture de Brossard oscille ainsi entre ce qu'on peut toucher (nos vies, ce qui respire, l'existence des autres, etc.) et ce qui doit demeurer intouchable (comme le silence). La vitesse et la lenteur, le réel et la fiction forment les contrepoints d'une prosodie souple et à laquelle fait directement allusion la seconde partie intitulée "Paumes flexibles". Vers ou prose, l'écriture brossardienne sait jouer des rythmes sans faire de fracas. D'autres, comme Roger Des Roches et Claude Beausoleil, pour ne citer que ceux-là, ont montré comment le poème pouvait et savait accueillir la prose qui vient donner aux vers leur élasticité et leur discrète désinvolture. Brossard userait quant à elle d'une écriture tranquille, à la pensée flexible; la poète a toujours tenté d'accorder la souplesse du phrasé à une démarche réflexive:

$$
\begin{aligned}
& \text { sera dit felix } \\
& \text { dans une autre langue } \\
& \text { objet de réflexion } \\
& \text { cascade de petites ampoules } \\
& \text { électriques } \\
& \text { sera la mer tout près sera } \\
& \text { point chatoyant au loin si fragile } \\
& \text { trace troublante navire (p. } 49) \\
& \text { luz là-bas sera nuit en forme de } \\
& \text { navions }
\end{aligned}
$$

Le vertige marque bien cette attraction entre le sens et les sens, qui laisse aussi bien place à l'oreille qu'à l'intellect. La grammaire y est lumineusement palpable:

\section{[...] en tenant des mots simples au creux de sa main, elle admirait la manière dont chaque femme en appuyant sa bouche sur le présent tenait à la vie, au fracas de lèvres sur les bas-reliefs de mémoire de ventre et de culture (p. 78)}

La poésie se tourne vers une phénoménologie qui tire profit des analogies entre la langue, la fiction et le corps.

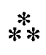

Les Tombeaux et ricocbets ${ }^{4}$ de Patrick Coppens ont une saveur autrement plus libertine, et par là, plus badine, que les chatoiements de la langue brossardienne. Le caractère double de l'intitulé invite aux associations paradoxales entre la profon- 
deur et la surface, le grave et le primesautier; ou si l'on comprend " tombeau " comme la forme littéraire d'un hommage posthume, il sera loisible d'y voir l'opposition entre la célébration et l'égratignure. Le choix de l'intitulé traduit bien le va-et-vient entre le discours tragique et le mot d'esprit, le ton sentencieux et les mignardises, entre le conte oniro-érotique de la Belle au bois dormant et les outrances d'un Barbe-Bleue plutôt bonhomme. Les textes versifiés alternent avec seize dessins de Mino Bonan, qu'il est difficile d'ignorer tant ils frappent par leur nombre et l'atmosphère étrange qui s'en dégage. En accord avec le ton du recueil, ils ont en commun de présenter une femme exposant le plus simplement du monde son intimité, ou se livrant à des actes sexuels auxquels elle ne semble prendre aucun plaisir. Le recueil s'ouvre et se referme comme un cercueil, au sein duquel l'énonciateur (Barbe-Bleue), reluqué par de petits êtres vicieux, enlace un cadavre de femme plus vive que morte:

Tombeaux et ricochets
j'enlace une morte impétueuse
les enfants d'Ézilie
accrochés au cercueil
avec des mains poisseuses
regardent d'un bon oeil
la morte s'amuser (p. 8 )

Les vers, fort brefs et fort ludiques (jeux de langue, jeux de vilains) composent une espèce de fête galante où la vie, la mort et l'amour, ce triangle inséparable, se retrouvent pour faire valser la Belle au bois dormant et l'horrible Barbe-Bleue. L'intitulé du recueil surgit à quelques reprises au beau milieu d'une séquence, comme un diablotin qui sort de sa boîte. Tout ceci n'est pas sérieux, nous dit le texte, et pourtant, ce l'est tout de même: tombeaux et ricochets. La déréliction a le dernier mot, ce qui n'est pas sans rappeler l'ironie grinçante de Jules Laforgue. Comme la lune face à Pierrot, la morte nargue le poète et ses velléités de rêverie amoureuse, elle "lui intime l'ordre / d'aller planter / ailleurs [s]es rêves" (p. 69). Ou ses choux. Malgré la vivacité des rythmes et des images, on danse à cloche-pied avec Patrick Coppens, et je crois bien que cela est dû à une surcharge dans le ludisme. Le clinquant des échos sonores, séduisant au premier abord, tourne un peu trop facilement à la facétie gratuite.

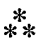

Yolande Villemaire s'est toujours intéressée au mythe, peut-être plus qu'à la fiction elle-même, qu'elle a su désamorcer par l'ironie. Sa poésie tellurique, qu'on pense à Quartz et mic $a^{5}$, montre des dispositions pour les discours des origines. Les murs de brouillard $^{6}$, par leur intitulé, annoncent déjà l'antinomie du roc et du monde plus poreux du rêve. Par le recours à un sujet féminin énoncé à la troisième personne, plus apte à traduire son entreprise épique, la poète effectue une remontée dans le temps pour retrouver la "femme aux ressources minérales" (p. 13). Interpellée par la culture indienne dans sa publication précédente ${ }^{7}$, celle-ci se lance cette fois à la recherche d'un autre savoir ancien, celui de la culture amérindienne, mais attention, celle qui remonte au-delà de ce que nous connaissons, et qu'on décèle uniquement aux traces qui viennent révéler un sujet féminin archaïque. Le 
recueil combine deux discours: d'un côté, la matérialisation de "[l]a femme du rêve" (p. 8), de l'autre, qu'on me pardonne ce chiasme facile, le rêve de la femme. Dans ce dernier cas, il s'agit d'un récit de rêve initiatique intitulé "L'attrapeur de rêve ${ }^{8}$ ", qui forme une suite de textes marqués par l'italique:

Des animaux de pouvoir paradent le long du sentier. Elle s'avance dans l'arborescence chthonienne, contourne des mares, escalade des rochers, éprouve l'élasticité de la mousse sous son pied nu. "la gratte de patte d'un petit lynx endormi, parfum de sa paume." (p. 40)

Le but de ce périple? Démanteler les murs de brouillard, à la suite de quoi "la langue de feu de la révélation" ( $\mathrm{p}$. 51) pourra prendre la forme du désir. Peut-on véritablement prendre au sérieux une poésie qui table sur l'inconnaissable et qui prend aussi peu de distance envers son propos? Ce qui sauvait le précédent recueil d'un parti pris trop exclusif de l'occulte, c'étaient les joyeuses correspondances entre la culture indienne et une petite enfance nourrie de catholicisme. L'idée de confier le discours uniquement à la nébuleuse du sacré oblige le lecteur à un acte de foi : on marche ou on ne marche pas. On a beau, comme Nerval, vouloir "pousser les portes d'ivoire et de corne ${ }^{9}$ ", il est des limites que même un lecteur bien disposé n'osera franchir. Ce que l'on peut regretter, c'est l'absence de distance ironique dont je parlais plus haut, qui permet à l'énonciateur de poser un regard amusé sur les choses en apparence les plus graves. Le mythe ne s'en porterait pas plus mal, bien au contraire.
Jean-Marc Lefebvre mise au contraire sur l'expression du sujet poétique: son recueil s'insère de plein pied dans la tradition lyrique. Apparemment peu attiré par la traversée des âges ou la migration des âmes, $L e$ chemin des vocables ${ }^{10}$ plante son décor dans le quotidien du réel. Chez Lefebvre, la fiction du sujet n'est jamais affirmée, comme c'est le cas chez Brossard, l'auteur affiche une assurance tranquille envers les recours de la poésie. Il marche dans la rue comme en lui-même et l'espace du poème, le poète insiste là-dessus, est intérieur. Nous sommes donc devant une métaphysique du quotidien, où le langage a pour fonction de produire "une pure présence" (p. 32). On croirait par instants entendre les voix de Pierre Morency ou de Jean Royer:

\section{Je déserte toute cause \\ qui ne parle pas \\ d'habiter son propre corps (p. 33)}

Au demeurant, la poésie de ce premier recueil est assez bien réussie, malgré la banalité qui semble prendre le dessus à mesure qu'on progresse dans la lecture. Mettant tous ses atouts dans "la posture essentielle / du regard" (p. 68), Le chemin des vocables, bien qu'il reste en deçà des modèles qu'il cite (Chamberland, Hénault, Garneau, et d'autres), mérite qu'on lui prête attention, notamment lorsqu'on s'arrête sur des passages d'une belle sobriété comme celui-ci :

La ville est calme nue jusqu'aux marges. Une lumière d'icône. Quelques brèches crues. Le reflet des choses ma main l'ignore (p. 43) 
Un recueil inégal, mais plein de promesses.

1. Nicole Brossard, Vertige de l'avant-scène, Trois-Rivières, Écrits des Forges/l'Orange bleue, 1997, $85 \mathrm{p}$.

2. Id., Langues obscures, Montréal, l'Hexagone, 1992, $59 \mathrm{p}$.

3. Id., Installations (avec et sans pronoms), Trois-Rivières/Pantin (France), Écrits des Forges/Le Castor astral, 1989, $125 \mathrm{p}$

4. Patrick Coppens, Tombeaux et ricochets (avec seize dessins de Mino Bonan), Montréal, Triptyque, 1997, $70 \mathrm{p}$.

5. Yolande Villemaire, Quartz et mica, TroisRivières/Pantin (France), Écrits des Forges/Le Castor astral, 1985, $54 \mathrm{p}$.
6. Yolande Villemaire, Les murs de brouillard, Trois-Rivières, Écrits des Forges, 1997, $51 \mathrm{p}$.

7. Yolande Villemaire, Lune indienne, TroisRivières, Écrits des Forges, 1994, 69 p.

8. Bien que cela ne soit pas dit dans le texte, je crois que cela fait référence à un objet magique amérindien plus connu sous la dénomination anglaise de "the dream catcher", objet qui avait effectivement la propriété d'attraper les rêves.

9. Gérard de Nerval, Aurélia ou le rêve et la vie, édition établie par Jean Richer, Paris, Rivard, coll. "Lettres modernes", 1965, p. 2.

10. Jean-Marc Lefebvre, Le chemin des vocables (tableaux de Marcel Saint-Pierre), Saint-Hippolyte, Le Noroît, 1997, 71 p. 